\title{
Low Resolution Face Recognition in Surveillance Systems
}

\author{
Xiang Xu, Wanquan Liu, Ling Li \\ Department of Computing, Curtin University, Perth, Australia. \\ Email: xiang.xu1@postgrad.curtin.edu.au
}

Received October 2013

\begin{abstract}
In surveillance systems, the captured facial images are often very small and different from the low-resolution images down-sampled from high-resolution facial images. They generally lead to low performance in face recognition. In this paper, we study specific scenarios of face recognition with surveillance cameras. Three important factors that influence face recognition performance are investigated: type of cameras, distance between the object and camera, and the resolution of the captured face images. Each factor is numerically investigated and analyzed in this paper. Based on these observations, a new approach is proposed for face recognition in real surveillance environment. For a raw video sequence captured by a surveillance camera, image pre-processing techniques are employed to remove the illumination variations for the enhancement of image quality. The face images are further improved through a novel face image super-resolution method. The proposed approach is proven to significantly improve the performance of face recognition as demonstrated by experiments.
\end{abstract}

\section{KEYWORDS}

\section{Face Recognition; Very Low Resolution; Surveillance Camera}

\section{Introduction}

Research in face recognition has been carried out for more than two decades, for its potentially wide applications in commercial and law enforcement fields. Popular face recognition approaches can achieve very high recognition performance in publicly released databases [1-6], where the resolution of the captured facial images is usually higher than $100 \times 100$. Some can even achieve similar high performance in a very low resolution [6], where the resolution of cropped faces is less than $10 \times 10$. However, most of these works have been conducted on databases where face images are captured in controlled environments with high definition cameras. The so-called "low-resolution" face images are derived from high-resolution faces through down-sampling and/or smoothing methods. When face images are captured directly in a "real" low resolution, the high performance of current face recognition approaches is yet to be proven.

Recently, face recognition research in real-life surveillance has become very popular. For high data transmission speed and easy data storage, surveillance cameras generally produce images in low resolution, and face images captured directly by surveillance cameras are usually very small. Besides, images taken by surveillance cameras are generally with noises and corruptions, due to the uncontrolled circumstances and distances. [7] proposed a super-resolution approach to increase the recognition performance for very low-resolution face images. They employ a minimum mean square error estimator to learn the relationship between low and high resolution training pairs. A further discriminative constraint is added to the learning approach using the class label information. [8] proposed a matching algorithm through using Multidimensional Scaling (MDS). In their approach both the low and high resolution training pairs are projected into a kernel space. Transformation relationship is then learned in the kernel space through iterative majorization algorithm, which is used to match the lowresolution test faces to the high-resolution gallery faces. Similarly, [9] proposed the Coupled Kernel Embedding approach, where they map the low and high resolution face images onto different kernel spaces and then transform them to a learned subspace for recognition.

Only a small portion of existing research is specifically for real surveillance scenarios, where the captured face images are very different compared with images captured under controlled circumstances. Most of the existing researches are based on the down-sampled low-resolution face images captured by high definition cameras under controlled environments. Even the works on surveillance cameras [7-10] on the claimed low-resolution (lower than $32 \times 32$ ) surveillance face images are in fact on images 
down-sampled from the original images captured in a resolution of $64 \times 64$. Face recognition based on true lowresolution (lower than $32 \times 32$ ) face images in uncontrolled surveillance scenarios remains an issue to be explored.

In this paper, we systematically analyze the key issues for face recognition in surveillance scenario, where the captured face images are usually with uncontrolled illumination, motion, poses and are generally taken in a far distance. Moreover, the off-the-shelf commercial surveillance cameras come with low-quality sensors and can only capture images in low resolutions.

Through our analysis, we found out that three factors which impact significantly on face recognition performances, including the distance between the camera and the human subject, types of cameras including sensor sizes and quality, and the resolutions of captured face images. Three experiments are designed to show the impact of these factors. We first demonstrate that the recognition performances on the low-resolution face images directly captured in real surveillance circumstances are much lower than those on the down-sampled low-resolution images from high-resolution images. It clearly indicates that the downsampled face images are not able to represent the true low-resolution images. By changing the types of cameras and the values of distances and resolutions, we demonstrate that face image resolution plays a key role in face recognition although the types of cameras and capturing distances are important factors.

Based on these observations, we propose an approach for face recognition in real surveillance environment. In this paper we focus on the indoor surveillance environment, e.g., in a corridor where people's motions are generally walking in a single direction in a relatively slow and steady pace. Our focus is hence on face recognition on surveillance captured face images with low resolutions, varied illumination conditions, small pose variation, and slow motions. Due to the very low resolution of the captured face images, many face features are lost. Image pre-processing ideas are employed to remove illumination variations as much as possible. In order to accumulate more features, we fuse a video sequence into one frame in the frequency domain. Curvelet features are adopted in the fusion process. The image is further improved through image super-resolution methods in order to increase the image resolution. Experimental results demonstrate that the proposed approach is able to improve the face recognition performance.

\section{Face Image Pre-Processing}

\subsection{Histogram Equalization for Illumination}

In real surveillance scenarios, directly captured low resolution images are different from those which are captured in controlled circumstances. Various factors influence the performance of face recognition, such as motion blur, and illumination and noises in images. In this paper we will focus on the surveillance of an ordinary indoor environment, where a normal range of illumination condition and distortion are considered without motion blur.

With a surveillance camera, video pictures are usually captured in low resolutions. The generic commercial surveillance cameras record pictures with resolutions varying from 400 to 800 pixels. For example the “SWANN DVR4-1300" commercial surveillance system used in CurtinFaces database [11] captures video sequences with the resolution of $576 \times 704$. While working in the indoor circumstance, the camera system captures very small faces in a distance. In the "SWANN DVR41300" commercial surveillance system, face resolutions are around $32 \times 32$ in the distance of approximately 2.5 meters, $16 \times 16$ in the distance of 5 meters and $8 \times 8$ in the distance of 10 meters respectively.

In an indoor corridor with no obvious side lighting, the face images captured demonstrate quite obvious illumination effects from the natural overhead lightings during a walking motion. A histogram equalization approach is adopted here for reducing illumination variations. There are generally two types of histogram equalization for image pre-processing [12]. One is the rank normalization where each pixel of the image is ranked and mapped to a new image between the values of 0 and 255. Another one is to pre-define a distribution of an image's pixels and re-map the image into the pre-defined model. Due to the similar feature on most part of face images, we adopt the second method in our approach.

In detail, for a $32 \times 32$ grey scale face image $\mathrm{x}$, the rank for each pixel is normalized to be $\mathrm{r}_{\mathrm{i}, \mathrm{j}}\left(\mathrm{r}_{\mathrm{i}, \mathrm{j}} \in[1,1024]\right)$ and the number of pixels is 1024 and the grey scale image level is 256. A general mapping function for pixel $x_{i, j}$ is defined as:

$$
p_{i, j}=\frac{1024-r_{i, j}+0.5}{1024}=\int_{x=-\infty}^{t_{i, j}} f(x) d x=F(x)
$$

where $t_{i, j}$ is the rank of pixel $x_{i, j}$ in the re-mapped space with distribution function $\mathrm{f}(\mathrm{x})$ and $\mathrm{F}(\mathrm{x})$ is the cumulative distribution function (CDF) for a given distribution $\mathrm{f}(\mathrm{x})$.

In order to remove the illumination variation, we assume that the intensity distribution of face images matches the standard normal distribution. The re-mapped face images can be derived from the inverse cumulative distribution function. For the pixel $\mathrm{x}_{\mathrm{i}, \mathrm{j}}$, the re-mapped rank $t_{i, j}$ is derived from:

$$
t_{i, j}=F^{-1}\left(p_{i, j}\right)
$$

where $F-1$ is the inverse cumulative distribution function and

$$
F(x)=\int_{-\infty}^{t} f(x) d x=\frac{1}{\sigma \sqrt{2 \pi}} \int_{x=-\infty}^{t} e^{-(x-\mu)^{2} / 2 \sigma^{2}} d x
$$


The grey scale face image after histogram equalization is derived through adjusting the pixel rank $t_{i, j}$ to the interval $[0,255]$.

\subsection{Fusion of Video Sequence}

Surveillance cameras usually capture whatever happens in a fixed environment into a video sequence. A set of images belong to one person with minor differences in poses and expressions can be extracted from the video. Illumination differences could be minimized after histogram equalization as described in the last section. In order to enhance the spectral features for face recognition, image fusion method [13] is adopted here. Generally there are two ways for image fusion. One is fusion in the spatial domain and the other is fusion in the frequency domain. In this paper, we utilize the Curvelet coefficients to represent the face features $[14,15]$.

For face recognition, Curvelet features have been proved to perform excellently in face feature representtation [16]. The proposed Curvelet based image fusion is represented in Figure 1 which indicates that several video frames can be fused into one image in order to derive rich features. It is expected to generate a face image which provides more features for face recognition. From [16] we can see that fine coefficients represent the character of a human better. For a sequence of facial images, we first transfer them into Curvelet Coefficients. The smallest low-frequency components which are represented by the coarse Curvelet coefficients and the biggest high-frequency components which are represented by the fine Curvelet coefficients are therefore kept in the proposed approach.

For the image sequence $I_{1}, I_{2}, \ldots, I_{n}$, their coefficients are represented as $C_{i}\{j\}\{l\}\left(k 1, k_{2}\right)(I=1,2, \ldots, n)$. The components of the first scale where $j=1$ represent the low-frequency parts of the face image and the components of other scales represent the high frequency parts. The minimum components between each $\mathrm{C}_{\mathrm{i}}\{\mathrm{j}\}\{\mathrm{l}\}(\mathrm{k} 1$, $\left.\mathrm{k}_{2}\right)(\mathrm{i}=1,2, \ldots, \mathrm{n})$ and the maximum components between each $C_{i}\{j\}\{l\}\left(k 1, k_{2}\right)(i=1,2, \ldots, n ; j \neq 1)$ are kept for the fused Curvet coefficients $C\{j\}\{l\}\left(\mathrm{k}_{1}, \mathrm{k}_{2}\right)$. After inverse Curvelet transformation, the fused face image can be derived as shown in Figure 1.

\section{Super-Resolution Based Face Recognition}

In real surveillance video sequences, face images taken beyond certain distance always come with noticeable noises and corruptions. When the captured face images are below $32 \times 32$, corruptions are obvious. Directly applying existing face recognition approaches on them generally will not achieve acceptable recognition performances. In order to enhance the face features, we propose a super-resolution based face recognition algo- rithm.

Inspired by [17], we make use of the sparsity of signal representation to train low-resolution image patches $\mathrm{p}_{\mathrm{l}}$ through a dictionary $\mathrm{Dl}$ and transfer the trained relationship onto the corresponding high-resolution dictionary Dh to reconstruct the high-resolution patch ph. This dictionary is trained in the FRGC [18] face database independently with both the high-resolution and low-resolution pairs. The high-resolution patch $\mathrm{p}_{\mathrm{h}}$ is reconstructed through adopting the same coefficients in the low-resolution training relationship, where a low-resolution patch $\mathrm{p}_{\mathrm{l}}$ is represented by a low-resolution dictionary $\mathrm{D}_{\mathrm{l}}$ with the relationship of $\alpha$. A high-resolution face image can be derived by combining all the high-resolution patches together. The low-resolution sparse representation is formulated as:

$$
\begin{aligned}
& \alpha \hat{\text { argmin }}\|\alpha\|_{1_{1}} \\
& \text { s.t. } \mathrm{D}_{1} \alpha=\mathrm{p}_{1}
\end{aligned}
$$

where $\alpha$ is the sparse representation coefficient $\sin l_{1}$ norm.

This sparse representation relationship is mapped to the high dimension space. The high-resolution image patch is derived from:

$$
P_{h}=D_{h} \alpha^{\wedge}
$$

After combing the two high-resolution patches, the hallucinated face image $y$ can be derived.

Meanwhile, we adopt the idea of [19] to enhance the same low-resolution face image into a high-resolution one. This process utilizes the Eigen-subspace features of human faces, which has been proved to have a good and stable performance in face feature representation [2]. For a set of training data (FRGC [18] in this paper), the covariance of zero mean face images $L$ is: $C=L \times L^{T}$. A zero mean low-resolution face image $x$ can be represented by the Eigenvectors Eas:

$$
x=E \times w+m
$$

where $w$ is the weight of Eigen faces and $m$ is the mean face. Equation (5) can be rewritten as:

$$
x=\left(L \times V \frac{1}{\sqrt{\Lambda}}\right) w+m=L \times \alpha+m
$$

where $\mathrm{V}$ is the Eigenvectors of covariance matrix $C=L^{T} \times L$ and $E=L \times V \frac{1}{\sqrt{\Lambda}}$.

The hig-resolution face y can be derived from:

$$
y=H \times \alpha+m_{h}
$$

where $H$ is the corresponding high-resolution training data of $L$ and $m_{h}$ is the high-resolution mean face.

After obtaining two high-resolution faces from the same low-resolution one, a decision is made for each pixel based on the low-resolution face image. For exam- 


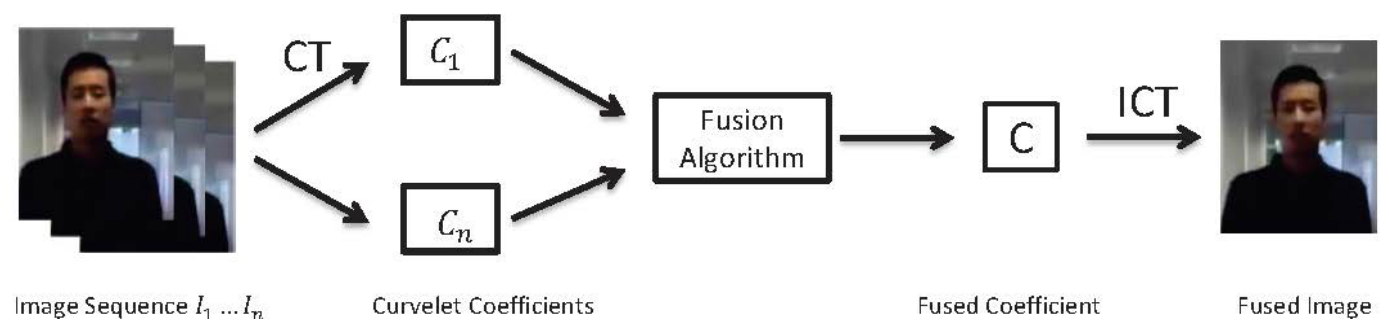

Figure 1. Image Fusion Process Diagram.

ple, for a $16 \times 16$ face image, we first enhance it into two high-resolution images using the methods described above. Both these high-resolution face images are then combined into one image with a pixel by pixel decision-making. For each pixel $x_{i, j}$ in the low-resolution image, the corresponding pixels in high-resolution is a $4 \times 4$ block. For a $16 \times 16$ low-resolution face, the reare 256 blocks in the high-resolution image. Assume the blocks from the two different enhanced face images are $b_{1}$ and $b_{2}$ respectively. In order to decide which block is to be kept, we down-sample both the $4 \times 4$ blocks into one pixel and keep the one which produces the pixel value closer to the value of the original low-resolution pixel $x_{i, j}$. The final enhanced block image is:

$$
\begin{aligned}
& \arg \min \operatorname{Down}(b)-x_{i, j} \\
& \text { s.t. } b=\lambda \times b_{1}+(1-\lambda) \times b_{2}
\end{aligned}
$$

where $\lambda$ equals to 0 or 1 .

After combing the 256 blocks together, the final enhanced face image is obtained which will be used for recognition.

\section{Experiments and Results}

In this paper, the experiments are performed on four databases: FRGC [18], AR [20], ScFace [10] and Curtin Faces [11]. FRGC and ARdatabases are captured with high definition cameras. Low-resolution images are down-sampled and smoothed from high-resolution ones. ScFace and Curtin Faces data bases contain face images from both high definition cameras and surveillance cameras. All the face images are cropped and aligned before being used. The high definition cameras used inAR, ScFace and Curtin Faces databases are SONY3CCDs, CanonEOS10D and PanasonicLumix respectively. The surveillance cameras used in ScFace database are: Bosch LTC0495/51, ShannyWTC-8342, ShannyMTC-L1438, JSJCC-915D and VFD400-12B. The surveillance camera used in Curtin Faces database is SWANNDVR4-1300.

In real world, the reare generally two reasons for a captured face image to be very small. One is that the distance between the camera and the person is too large and the other is that the camera sensor is limited. Although the focal length of a camera can always be changed, when the distance between a camera and an object is too far away, the captured images become very small. For simplicity, we assume that all cameras in our experiments have fixed focal length.

In our experiments, the resolutions of face images are the originally captured sizes unless specified otherwise. None of the images are down-sampled from high resolution images. For simplicity, we divide face image resolutions into five levels: $128 \times 128,64 \times 64,32 \times 32,16 \times$ 16 and $8 \times 8$. The face images are directly cropped from the surveillance images, and if the cropped images are not exactly the desired sizes, they are slightly changed through Cubic interpolation to the nearest resolution level. Four experiments are conducted. Experiment 1 compares recognition performances between two different types of low resolution image. One type is directly captured with large distance between the camera and the person. Another type is from down-sampling from high-resolution images. Results from Experiment 1 demonstrate that the recognition performances for the directly captured images are much lower than the low-resolution images. In Experiment 2 the distance between the camera and the person is fixed. It compares the recognition performances between different types of cameras, resulting in different resolutions in the captured images. In Experiment 3: the image resolution is fixed. Recognition performances are compared on face images from various sources, whereas the types of camera and capturing distances vary. The recognition performance of our proposed approach on surveillance face images is demonstrated in Experiment4.

\subsection{Down-Sampling vs Captured Low Resolution}

Lots of work has been done on low-resolution face recognition. However, most of the existing works are on low-resolution face images down-sampled from highresolution images. In real life, most low-resolutions are due to the large distances between the cameras and the face. It is hence worthwhile to evaluate whether the down-sampled images provide a good representation of the true low-resolution images. Here we compare the recognition difference between the down-sampled images and images captured by cameras in a far distance. Face recognition is first performed on images from the popular AR database. Figure 2(a) shows the recognition 
rates in terms of different down-sampled resolutions on AR database. In this experiment, we randomly select 13out the 26 images per person for training and the other 13 for testing. This procedure is repeated 10 times to obtain the average recognition rate. Similarly, face recognition results on the Curtin Faces High Definition database are shown in Figure 2(b). Here, only 25 images are selected per person from the available 92 images among which images with large pose and illumination variations are excluded. 12 images out of the 25 are randomly selected for training and the other 13 are for testing. It can be seen from these two figures that when lowresolution face images are down-sampled from highresolution ones, their recognition rates do not reduced much. Even very low-resolution $(8 \times 8)$ faces can still achieve a satisfactory recognition rate (around $90 \%$ ).

However, when image resolution drops due to the increased distances, recognition rates decrease very sharply, as shown in Figure 2(c). In this figure, face images are captured using the same High Definition camera as in $b$.

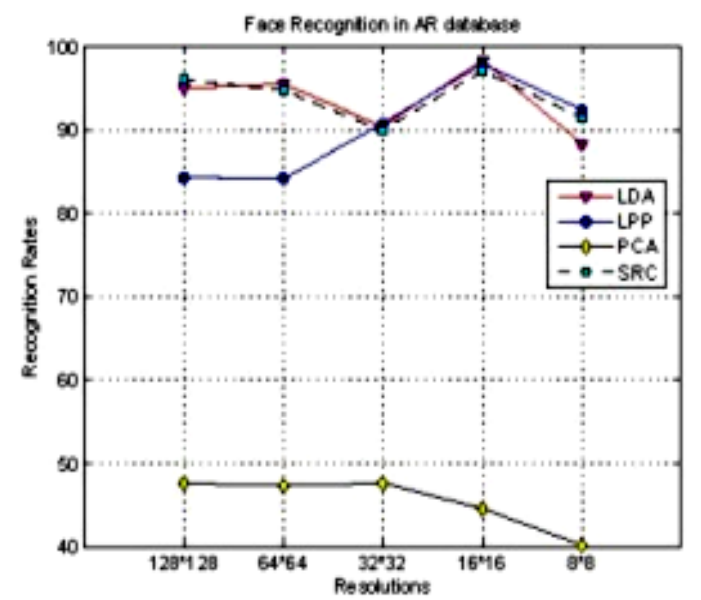

(a)

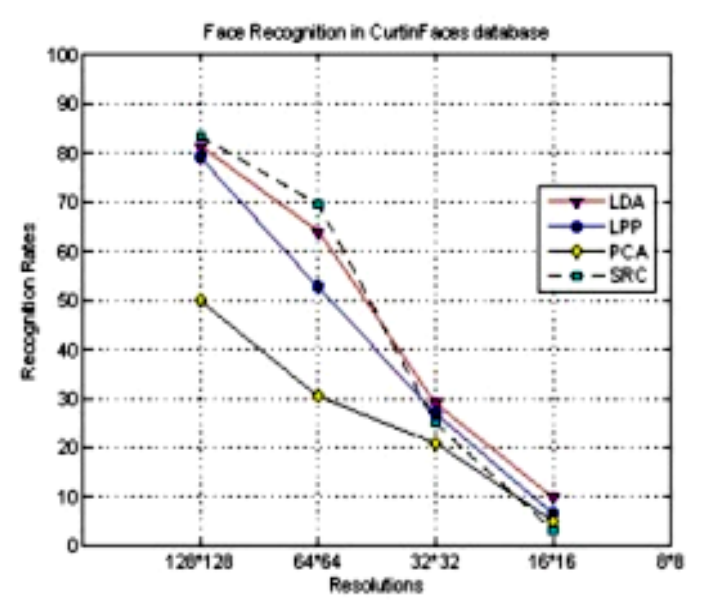

(c)
Instead of down-sampling images to low-resolution, images in this figure are captured from various distances in the same environment. The resolutions of the captured face images are approximately in the resolution levels of $128 \times 128,64 \times 64,32 \times 32$, and $16 \times 16$ in the distances of 2.5 meters, 5meters, 10meters and 20meters respectively. In clear contrary to the various resolutions from down-sampling, decreasing of resolutions due to the increased distance from camera caused the recognition rates drop very sharply as shown in Figure 2(c).

It can be concluded that the down-sampled face images are not good representations of captured low-resolution images for face recognition. Face recognition performance with directly captured images in distances through High Definition cameras is very low.

To further demonstrate the difference between downsampling and distance sampling, databases captured through surveillance cameras are adopted. Figure 2(d) and Table 1 show the face recognition rates in Curtin Faces Surveillance Camera database and ScFace database

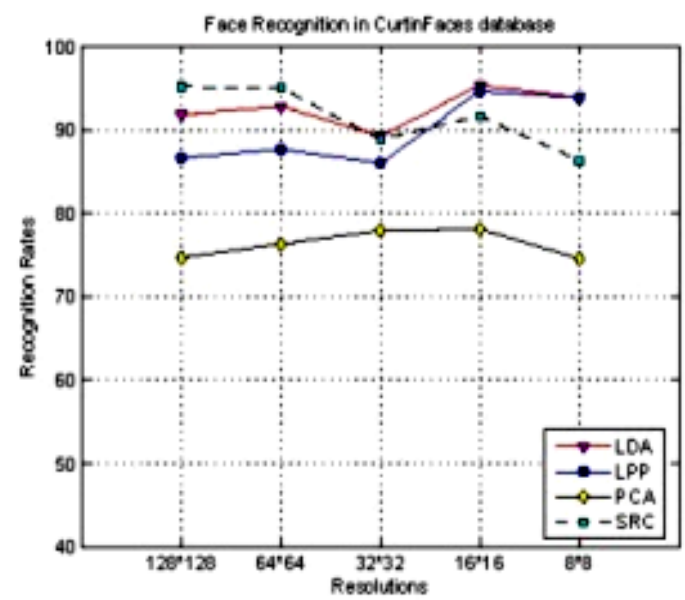

(b)

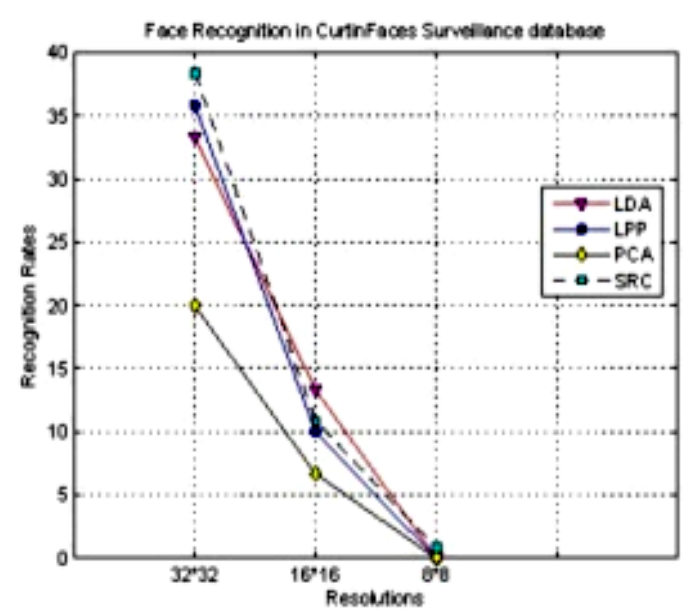

(d)

Figure 2. Down-sampling vs distance sampling. 
Table 1. Face recognition performance in scface database.

\begin{tabular}{lcccc}
\hline & LDA & LPP & PCA & SRC \\
\hline Camera 1 & 3.08 & 4.62 & 13.85 & 13.85 \\
Camera 2 & 5.38 & 4.62 & 18.46 & 14.62 \\
Camera 3 & 3.85 & 4.62 & 16.42 & 10.00 \\
Camera 4 & 1.54 & 7.69 & 20.77 & 12.31 \\
Camera 5 & 3.08 & 6.15 & 12.31 & 3.08 \\
\hline
\end{tabular}

respectively. The face images of the mare captured with different surveillance cameras in far distances. It can be seen that regardless of different recognition approaches, the recognition rates are also very low when images are captured in distances instead of down-sampled from high-resolution images through surveillance cameras.

\subsection{High Definition Cameras vs Surveillance Camera}

It has been shown in Figure 2(c) that even images captured from a high definition camera are unable to warrant a good recognition performance. In this experiment we evaluate the performance of surveillance camera in indoor surveillance scenarios. The Curtin Faces Surv database contains video sequences from a surveillance camera which captures human faces in the same environment as the high definition camera used above. The surveillance camera is a commercial video surveillance camera with the image resolution of $704 \times 576$. The original resolutions of the cropped face images from the surveillance camera are approximately $32 \times 32,16 \times 16$ and $8 \times 8$ taken in the distances of 2.5meters, 5meters and 10 meters respectively. Face recognition performance by the popular LDA, LPP, PCA and SRC methods are shown in Figure 2(d). The recognition rates can be observed to be similar to those of the high definition cameras with different distances (Figure 2(c)). However, when the distances are fixed, e.g., in 5meters, the differences of cameras and resolutions lead to huge differences in recognition rates. In this distance the SRC recognition rate for high definition camera is around $70 \%$ with the resolution $64 \times 64$, while the SRC recognition rate for surveillance camera is around only $11 \%$ with the resolution $16 \times 16$.

\subsection{Cameras, Distance and Resolution}

This experiment aims to explore the influences of the types of cameras, distances and resolutions for surveilance face recognition. From Experiment 1, we can seethat when the same camera is used, images taken in different distances result in totally different recognition performance. As shown in Experiment 2, when the distance is fixed, images taken by different cameras have large differences in the recognition performance. What would a fixed resolution lead to? We select two different resolutions in this experiment. Figure 3(a) shows the recognition performance for images with the resolution of $16 \times 16$. Images with this resolution are captured by the high definition camera at the distance of 20 meters and by the surveillance camera only from 5 meters away. Figure 3(b) shows the performance in the resolution of $32 \times 32$, where HD camera is at a distance of 10 meters and surveillance camera is at a distance of 5 meters. We can see from both figures that despite the differences in camera types and shooting distances, face images with same resolutions result in similar recognition performances.

\subsection{Face Recognition by Super Resolution}

In this experiment, the proposed face recognition method is applied and tested. Here, we carry out the experiment on the surveillance camera. Figure 4(a) demonstrates the recognition performance comparison between the captured faces in the distance of 5 meters by the surveillance camera and the enhanced images by the proposed approach. In this setting, the original face resolution is $16 \times$ 16 and the enhanced face resolution is $64 \times 64$. Figure 4(b) shows the recognition performance comparison between the captured faces in the distance of 10 meters by the HD camera and the enhanced faces by the proposed approach. As shown in Figure 4, the face recognition rates are greatly improved after the images are processed using the proposed method, no matter which recognition method is used.

\section{Conclusion}

Avoid Traditional face recognition approaches can hardly achieve satisfactory performance on low-resolution images, esp. on those directly captured by surveillance cameras. Till now little work has been done specifically on face recognition based on surveillance cameras. In this paper, we analyze the factors which impact on face recognition performances in surveillance scenario. Experiments indicate that other than camera types and capturing distances, image resolution is the major factor affecting the performance of face recognition in surveillance circumstance. Specifically we can conclude that the higher resolution the images, the better performance face recognition achieves.

According to the special conditions of a surveillance system, we proposed a super-resolution based face recognition approach. Experiments demonstrate that our approach outperforms traditional face recognition approaches significantly.

Although the proposed approach performs well for very low resolution face recognition in surveillance system, more practical surveillance conditions need to beconsidered, such as motion blur, extremely low resolu- 


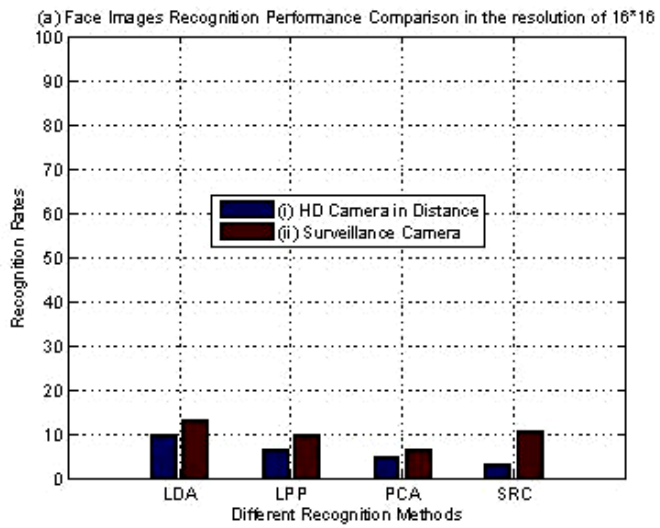

(a)

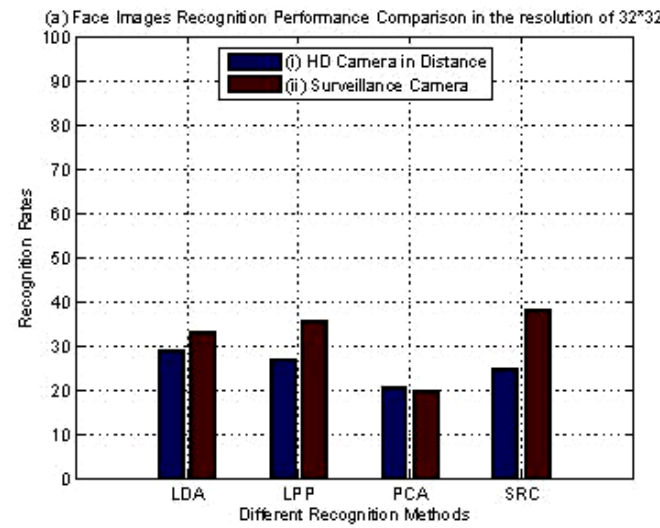

(b)

Figure 3. Recongnition comparision with the same resolution.

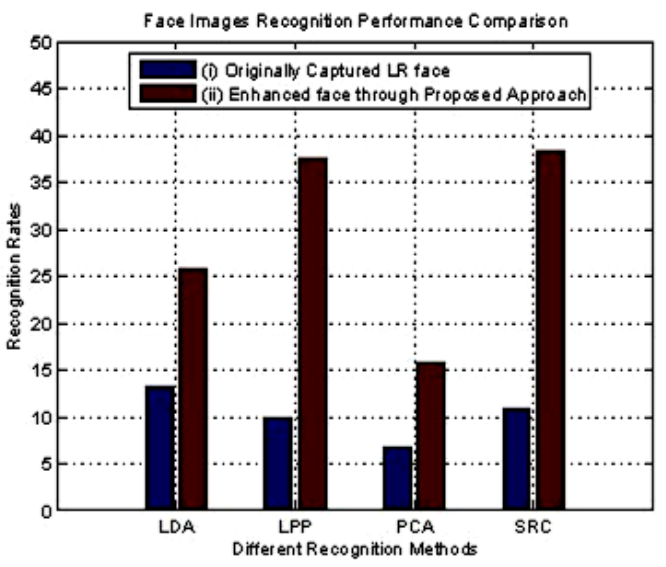

(a)

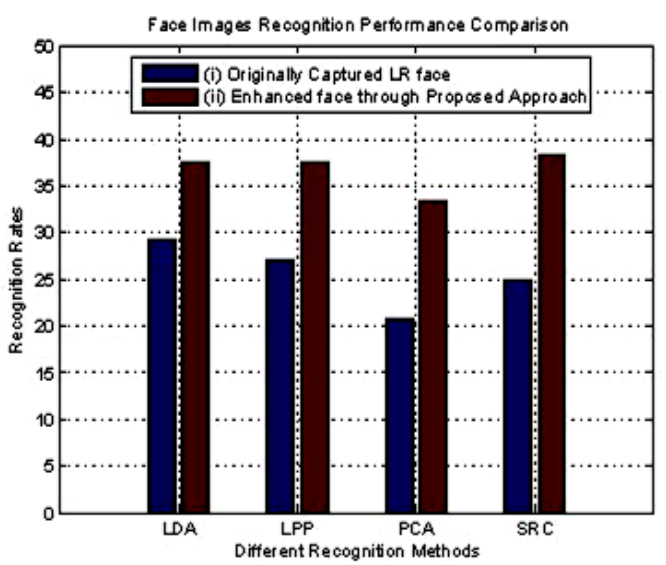

(b)

Figure 4. Recognition performance comparision between originally captured face images and proposed approach.

tion (less than $10 \times 10$ ) and face recognition in outdoor conditions and from very far distances. They will be our future work.

\section{REFERENCES}

[1] W. Zhao, et al., "Face Recognition: A Literature Survey," ACM Computing Surveys, Vol. 35, 2003, pp. 399-458.

[2] M. A. Turk and A. P. Pentland, "Face Recognition Using Eigenfaces," IEEE Computer Society Conference on Computer Vision and Pattern Recognition, 1991, pp. 586591.

[3] P. N. Belhumeur, J. P. Hespanha and D. J. Kriegman, "Eigenfaces vs. Fisherfaces: Recognition Using Class Specific Linear Projection,” IEEE Transactions on Pattern Analysis and Machine Intelligence, Vol. 19, 1997, pp. 711-720.

[4] X. He and P. Niyogi, "Locality Preserving Projections," Advances in Neural Information Processing Systems, Vol. 16, 2004, pp. 153-160.

[5] A. S. Georghiades, P. N. Belhumeur and D. J. Kriegman,
"From Few to Many: Illumination Cone Models for Face Recognition under Variable Lighting and Pose," IEEE Transactions on Pattern Analysis and Machine Intelligence, Vol. 23, 2001, pp. 643-660.

[6] J. Wright, et al., "Robust Face Recognition via Sparse Representation," IEEE Transactions on Pattern Analysis and Machine Intelligence, Vol. 31, 2009, pp. 210-227.

[7] W. W. W. Zou and P. C. Yuen, "Very Low Resolution Face Recognition Problem," IEEE Transactions on Image Processing, Vol. 21, 2012, pp. 327-340.

[8] S. Biswas, K. W. Bowyer and P. J. Flynn, "Multidimensional Scaling for Matching Low-Resolution Face Images," IEEE Transactions on Pattern Analysis and Machine Intelligence, Vol. 34, 2012, pp. 2019-2030.

[9] C.-X. Ren, D.-Q. Dai and H. Yan, "Coupled Kernel Embedding for Low-Resolution Face Image Recognition," IEEE Transactions on Image Processing, Vol. 21, 2012, pp. 3770-3783.

[10] M. Grgic, K. Delac and S. Grgic, "SCface-Surveillance Cameras Face Database,” Multimedia Tools and Applications, Vol. 51, 2011, pp. 863-879.

[11] B. Y. L. Li, et al., "Using Kinect for Face Recognition 
under Varying Poses, Expressions, Illumination and Disguise," IEEE Workshop on Applications of Computer Vision (WACV), 2013, pp. 186-192.

[12] V. Štruc, J. Žibert and N. Pavešić, "Histogram Remapping as a Preprocessing Step for Robust Face Recognition,” Image, Vol. 7, 2009, p. 9.

[13] H. B. Mitchell, "Image Fusion: Theories, Techniques and Applications,” 2010.

[14] E. Candes, et al., "Fast Discrete Curvelet Transforms," Multiscale Modeling Simulation, 2006.

[15] E. J. Candès and D. J. Donoho, “Curvelet: A Surprising Effective Non-Adaptive Representation for Objects with Edges,” Department of Statistics, 1999.

[16] T. Mandal, Q. M. J. Wu and Y. Yuan, "Curvelet Based
Face Recognition via Dimension Reduction,” Signal Processing, Vol. 89, 2009, pp. 2345-2353.

[17] J. Yang, et al., "Image Super-Resolution via Sparse Representation," IEEE Transactions on Image Processing, Vol. 19, 2010, pp. 2861-2873.

[18] P. J. Phillips, et al., "Preliminary Face Recognition Grand Challenge Results," 7th International Conference on Automatic Face and Gesture Recognition, 2006, pp. 15-24.

[19] X. Wang and X. Tang, "Hallucinating Face by Eigentransformation," IEEE Transactions on Systems, Man, and Cybernetics, Vol. 35, 2005, pp. 425-434.

[20] A. M. Martinez and R. Benavente, "The $\{A R\}$ Face Database,” CVC Technical Report, 1998. 